\title{
INTEROPERABILIDAD Y POLÍTICA SANITARIA EN ESPAÑA. EL CASO DE LA HISTORIA CLÍNICA DIGITAL DESDE UNA PERSPECTIVA INTERGUBERNAMENTAL
}

\author{
INTEROPERABILITY AND HEALTH POLICY IN SPAIN. THE CASE OF DIGITAL \\ MEDICAL RECORDS FROM THE INTERGOVERNMENTAL PERSPECTIVE
}

\author{
J. Ignacio Criado Grande \\ Universidad Autónoma, Madrid. España/Spain \\ ignacio.criado@uam.es
}

Recibido/Received: 22/03/2013

Modificado/Modified: 31/05/2013

Aceptado/Accepted: 20/06/2013

\section{RESUMEN}

Diferentes proyectos de interoperabilidad se han desarrollado durante los últimos años en todo el mundo. La interoperabilidad en la Administración electrónica es un facilitador clave para compartir información y conocimiento, y resulta también necesaria para cumplir algunas de las promesas derivadas del uso de las Tecnologías de las Información y la Comunicación (TIC) en el sector público. Este artículo presenta una investigación los resultados preliminares de una investigación sobre la interoperabilidad de la política sanitaria en España, con una atención especial a los niveles de gobierno central y autonómico, que cuentan con las competencias para su regulación y gestión. Este estudio tiene como objetivo perfilar cómo se han desarrollado las categorías de interoperabilidad técnica, semántica y organizativa, además de la gobernanza de la interoperabilidad en las relaciones entre gobierno central y autonómico. Para ello, se presenta un proyecto intergubernamental dentro del ámbito sanitario español, la Historia Clínica Digital del Sistema Nacional de Salud, que ha logrado un elevado nivel de desarrollo, no si dificultades y tras un extenso período de definición e implementación. Lo anterior pretende arrojar luz y abrir la discusión en torno a los retos y oportunidades de la aplicación de un sistema de gobernanza multinivel de las TIC en el ámbito de la política sanitaria española.

\section{PALABRAS CLAVE}

Relaciones intergubernamentales, salud, historia clínica, estudio de caso.

\section{SUMARIO}

1. Introducción. 2. Aspectos generales sobre interoperabilidad en el sector público. 3. Interoperabilidad en el ámbito sanitario español. 4. Estudio de caso. La Historia Clínica Digital del Sistema Nacional de Salud. 5. Discusión y conclusiones. 6. Agradecimientos. Bibliografía.

\footnotetext{
ABSTRACT

During the last years, different interoperability projects have been undertaken around the world. Interoperability of Electronic Government is an enabler to share information and knowledge, and it is essential to fulfill some of the promises of Information and Communication Technologies in the public sector. This article presents the preliminary results of a research about interoperability of the health policy in Spain, regarding to the central and regional levels of government. They share the competences of regulation and management of this policy sector. Also, this study will draw the evolution of the
} 
technical, semantic, and organizational interoperability dimensions, together with the governance system, with special attention to central and regional authorities' relations. In so doing, we present the case of the Electronic Records of the National Health System, that it is one of the most developed projects in the field of interoperability in Spain. This analysis of this case study is devoted to shed light on the new challenges and opportunities for the application of a multi-level governance system fostering ICTs in the health policy in Spain.

\section{KEYWORDS}

Inter-governmental relations, health, clinical records, case study.

\section{CONTENTS}

1. Introduction. 2. General aspects of interoperability in the public sector. 3. Interoperability in Spanish healthcare. 4. Case Study. 5. Discussion and conclusions. 6. Acknowledgements. References.

\section{INTRODUCCIÓN}

Este artículo presenta un estudio sobre la interoperabilidad en el ámbito sanitario español. En concreto, trata de abrir una discusión derivada de un análisis del proceso de interoperabilidad que se está llevando a cabo en esta política pública, teniendo en cuenta las relaciones intergubernamentales entre la Administración General del Estado y las administraciones de las comunidades autónomas en España. En particular, se analizará el proyecto de Historia Clínica Digital del Sistema Nacional de Salud, en la medida que representa de una manera coherente las diferentes dimensiones (técnica, semántica, organizativa y de gobernanza) que son necesarias para llevar a cabo una iniciativa de esta naturaleza. Todo lo anterior delimita un espacio ideal para ofrecer una serie de conclusiones ligadas a las administraciones públicas iberoamericanas y las necesidades de interoperabilidad crecientes dentro de la región (Criado, et al., 2011).

La interoperabilidad, entendida al menos como la capacidad mediante la cual sistemas heterogéneos, no sólo tecnológicos, pueden intercambiar información y procesos técnicos o datos, no es algo completamente nuevo dentro del sector público. Sin embargo, cada vez más, toma este término mayor importancia entre las administraciones públicas como consecuencia de la necesidad de satisfacer mejor las necesidades y expectativas del ciudadano y, por tanto, de implantar proyectos de Administración electrónica (eAdministración) más sofisticados y complejos que requieren la colaboración de dos o más órganos administrativos (Criado, et al., 2011; Gascó y Roy, 2006; Gil-García, et al., 2007; Mayer-Schönberger, 2005; Scholl y Klischewski, 2007). En el ámbito sanitario los requerimientos de colaboración entre diferentes entidades son todavía más importantes, especialmente, si se desea ofrece una atención de la máxima calidad.

En este artículo se pone el acento en la necesidad proceder a una adecuada gobernanza de los procesos de interoperabilidad dentro de las administraciones públicas. Como ya se ha puesto de manifiesto en otros trabajos previos (Criado, 2009; Dos Santos, 2008; Gascó, 2010), la interoperabilidad no es una cuestión que se circunscriba a la dimensión técnica, sino que en realidad se ha de sustentar en una dimensión política (gobernanza), que suele marcar la diferencia en cuanto a los resultados de los proyectos planteados. Por ello, el presente trabajo pretende dotar a esa dimensión de un interés singular, sin descuidar las otras vertientes de la interoperabilidad. Así, el análisis realizado sobre el caso de la 
Historia Clínica Digital en el Sistema Nacional de Salud español se fundamenta en una revisión documental de toda la información disponible acerca del mismo, tratando de caracterizar las líneas estratégicas más relevantes y la forma de la gobernanza presente en su implementación.

Este documento se estructura de la siguiente manera. En la siguiente sección se presentan los aspectos generales sobre la interoperabilidad en el sector público, ofreciendo una definición, resumiendo sus beneficios, así como identificando los tipos y niveles de interoperabilidad. A continuación, se hace una consideración específica sobre la interoperabilidad en la política sanitaria española. En la cuarta sección se describe y analiza el caso de la Historia Clínica Digital en el Sistema Nacional de Salud. Finalmente, se plantea una discusión de cara a ofrecer ideas de interés teniendo en cuenta el contexto de las administraciones públicas españolas, así como unas conclusiones de futuro en torno a esta cuestión.

\section{ASPECTOS GENERALES SOBRE INTEROPERABILIDAD EN EL SECTOR PÚBLICO}

\subsection{Concepto de interoperabilidad}

Desde las instituciones de normalización internacionales se ha realizado un esfuerzo por definir interoperabilidad, si bien desde una perspectiva limitada a datos e información, y pensando en su aprovechamiento para el sector privado:

"Habilidad de dos o más redes, sistemas, aplicaciones, dispositivos o componentes para intercambiar información entre ellos y usar esa información intercambiada". (CEN, CENELEC, ETSI y ANSI, agencias europeas y norteamericanas de estandarización, Conferencia sobre Interoperability: Key to International Business, celebrada en Varsovia, Polonia, 6-7 Febrero, 2008)

El gobierno australiano fue uno de los pioneros en plantear la problemática de la interoperabilidad, concretando su aproximación de la siguiente manera, en línea con lo anterior:

"Habilidad de transferir y utilizar información de manera uniforme y suficiente entre varias organizaciones y sistemas de información". (Gobierno de Australia, 2006)

Desde las instituciones de la Unión Europea se puso de manifiesto también con mucha rapidez la necesidad de establecer un marco común de interoperabilidad, de manera que aquí se planteó lo siguiente:

"Capacidad de los sistemas basados en el uso de las TIC y de los procesos de negocio o gobierno en que se basan para intercambiar datos y permitir la puesta en común de información y conocimiento". (IDABC, 2004:5)

De manera más concreta, también se puede ofrecer una noción de lo que es interoperabilidad en el ámbito de la e-Salud. En concreto, la Unión Europea plantea lo siguiente:

"Capacidad de los sistemas de información sanitarios independientes y heterogéneos para intercambiar datos relacionados con la salud para uso de los médicos, proveedores y pacientes". (European Commission, 2012)

Todas estas definiciones ponen de manifiesto la relevancia de la normalización técnica y el principio de la cooperación administrativa como sustrato de la interoperabilidad. Una visión reduccionista tiende a considerar la interoperabilidad como un proceso de 
normalización de patrones tecnológicos o de TIC utilizadas en diferentes administraciones públicas. Sin embargo, eso es sólo el paso inicial para definir también una serie de pautas comunes mediante las cuales se consiga que las organizaciones públicas y sus sistemas de información interaccionen unos con otros. En otras palabras, hay que conseguir que los procedimientos, las formas de trabajar, las tecnologías y sistemas de información que anidan en cada administración no sean un obstáculo para interrelacionarse.

\subsection{Beneficios de la interoperabilidad}

Lo importante de las definiciones de interoperabilidad es que comparten una serie de aspectos en común que, sobre todo, apuntan a una serie de beneficios compartidos para todos los agentes involucrados. De lo anterior se deduce que los principales beneficios de la interoperabilidad dentro del sector público son algunos de los siguientes:

i. Pre-requisito a la puesta en funcionamiento de servicios públicos electrónicos interadministrativos y de alto valor añadido para el ciudadano, dado que se pueden involucrar diferentes agencias, independientemente del ámbito de que se trate.

ii. Cooperación entre niveles de gobierno y agencias de la administración sin distinción según el desarrollo tecnológico, esto es, combinando diferentes tipos de tecnologías en los back-office de las agencias involucradas.

iii. Simplificación de la actividad administrativa y de los procesos de negocio/gobierno.

iv. Posibilidad de utilizar más fácilmente estándares abiertos y aplicaciones tecnológicas comunes, así como la reutilización de componentes.

v. Armonizar las garantías de la seguridad, privacidad y derecho de acceso de los ciudadanos.

vi. Mayor accesibilidad y sencillez para el ciudadano y los actores que interactúan con el sector público.

vii. Capacidad de promover una mayor transparencia y rendición de cuentas ante la ciudadanía.

viii. Reusabilidad, a través del establecimiento de procesos y modelos similares para procesos paralelos.

ix. Explorar la cooperación transfronteriza a la hora de prestar determinados servicios o realizar determinadas actividades de apoyo que requieren el concurso de diferentes países para su implementación.

\subsection{Tipos de interoperabilidad}

La interoperabilidad no es sólo una cuestión tecnológica. La capacidad para intercambiar datos y permitir la puesta en común de información y conocimiento no se refiere exclusivamente a la integración de redes, no hace referencia sólo al intercambio de datos entre sistemas, ni contempla simplemente una definición de tecnología. Se trata de una adición de todos esos factores considerando la existencia de legados de sistemas, de plataformas hardware y software instalados, es decir, parte de principios que tratan de la diversidad de componentes con la utilización de diversos productos de múltiples proveedores en diferentes organizaciones. Pero además, tiene como objetivo la definición de políticas, normas y estándares para la consecución de la cooperación entre los sistemas de diferentes organizaciones públicas (Guijarro, 2007). Este último aspecto implica la preocupación por el funcionamiento de los procesos de negocio o procesos de gobierno, como requisito previo a las soluciones técnicas. En ese plano, desde la UE se han 
diferenciado tres vertientes de la interoperabilidad en el ámbito de la $e$ Administración que permite integrar las diferentes dimensiones del fenómeno (IDABC, 2004) (ver Figura 1):

Figura 1. Tipos de interoperabilidad

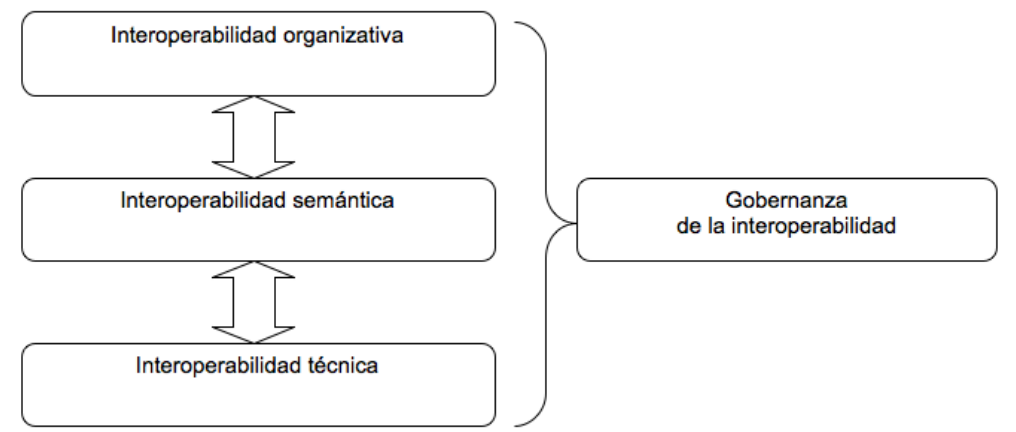

Fuente: adaptado de Comisión Europea (2007)

* Interoperabilidad técnica. La interoperabilidad tecnológica se refiere a todas las cuestiones técnicas (tecnologías, estándares, etc.) para garantizar que los componentes tecnológicos de los sistemas de información de las entidades participantes estén preparados para colaborar todos juntos. En este sentido, hay que insistir en que la interoperabilidad tecnológica no se restringe a las tecnologías dentro de la capa física de conexión (como por ejemplo los protocolos de red), sino que también abarca las tecnologías que ofrecen soporte a las capas organizativa y semántica.

* Interoperabilidad semántica. La semántica se refiere al significado en el uso de los datos y la información. Este tipo de interoperabilidad implica asegurar que el significado preciso de la información sea comprendido adecuadamente por otra aplicación que no fue desarrollada con su mismo propósito, es decir, que habilita a los sistemas para combinar la información proveniente de otras fuentes y procesarla de una manera integrada y con el sentido correcto. Por lo tanto, la interoperabilidad semántica constituye un prerrequisito para una integración de los servicios, en diversas lenguas, y su puesta a disposición para el usuario final.

* Interoperabilidad organizativa. Este ámbito se refiere a la definición de los objetivos de los procesos y servicios de las organizaciones implicadas en la prestación de servicios telemáticos. En concreto, la interoperabilidad organizativa hace referencia a la colaboración de organizaciones que desean intercambiar información manteniendo diferentes estructuras internas de gobierno y procesos de negocio variados. El objetivo de la interoperabilidad organizativa es superar los obstáculos organizativos, así como ser capaz de integrar los flujos de procesos inter e intra-organizativamente.

Más recientemente, se ha empezado a hablar de la necesidad de establecer una estructura de gobernanza de la interoperabilidad. En concreto, esta cuestión se utiliza para identificar los acuerdos entre gobiernos y actores que participan en los procesos de interoperabilidad y la forma de alcanzarlos, incluyendo los espacios de diálogo donde se definan los acuerdos. El propósito aquí es identificar y remover las potenciales barreras, incluyendo las legislativas, culturales y otras para agregar los servicios públicos y compartir información en contextos intergubernamentales. Un escenario para ejemplificar lo anterior sería aquel en el que varias instituciones públicas desean colaborar con el 
objetivo de agregar sus servicios telemáticos para mejorar la atención de las necesidades de la ciudadanía, por ejemplo, extendiendo entre ellas un enfoque centrado en hechos vitales: nacer, cambiar de domicilio, casarse, tener hijos, ir al médico, cambiar de trabajo, etc.

\subsection{Niveles de interoperabilidad}

Junto con el ámbito funcional, también se pueden establecer diferentes niveles de interoperabilidad. Varios niveles de interoperabilidad se identifican ligados a los tipos de actores públicos que se implican en los intercambios, de manera que los tres niveles se pueden considerar como interoperabilidad nacional o dentro de un mismo país, a diferencia del último de los casos de la presente tipología.

* Interoperabilidad intra-administrativa. Se trata de la que se produce dentro de una misma autoridad administrativa o gubernamental, pero entre diferentes departamentos o agencias pertenecientes al mismo.

* Interoperabilidad horizontal. Se refiere a aquella que se desarrolla entre diferentes administraciones dentro de un mismo nivel de gobierno (administración localadministración local; administración regional-administración regional).

* Interoperabilidad vertical. Se lleva a efecto cuando intervienen diferentes niveles de gobierno dentro de un mismo país (administración central-administración regionaladministración local).

* Interoperabilidad regional o transfronteriza. Se considera a la que tiene lugar cuando participan agencias o administraciones de diferentes países, independientemente del tipo de niveles de gobierno involucrados, si bien aquí lo habitual es que se trate de autoridades de gobiernos centrales o nacionales.

\section{INTEROPERABILIDAD EN EL ÁMBITO SANITARIO ESPAÑOL}

Uno de los ámbitos en los que la interoperabilidad ha estado más presente dentro del caso español se refiere a la política sanitaria. Sin entrar en el proceso de descentralización territorial del poder en el ámbito sanitario, lo que sí se puede decir es que cuando se completa el proceso de transferencias sanitarias a las regiones en el año 2001, uno de los problemas clave que se plantea entonces es cómo asegurar la cohesión del sistema. Por consiguiente, la política sanitaria española entra en una fase en las relaciones entre el nivel central de gobierno y las administraciones autonómicas que se convierte en decisivo, de manera que las TIC entran también a formar parte de esa dinámica intergubernamental.

\subsection{Aspectos organizativos y de gobernanza}

Desde un plano organizativo, la entidad que articula las relaciones entre el Ministerio de Sanidad y los departamentos autonómicos encargados de la sanidad es el Consejo Interterritorial del Sistema Nacional de Salud (CISNS). Este organismo intergubernamental, que facilita las relaciones entre niveles administrativos se creó en 1987, cuenta con representantes de la Administración General del Estado (responsable del ministerio) y de las comunidades autónomas (responsables de las consejerías autonómicas y ciudades autónomas). Los acuerdos del CISNS se plasman a través de recomendaciones que se aprueban, en su caso, por consenso, de manera que los acuerdos de cooperación para llevar a cabo acciones sanitarias conjuntas se formalizan mediante convenios. 
En términos operativos, el CISNS ha desarrollado una intensa labor que merece la pena considerar. En CISNS es unos de los instrumentos multilaterales en España que más veces se ha reunido desde su creación (96 reuniones) (SEAP, 2012). Además, su actividad se ha ido intensificando durante los últimos años de una forma notoria. La media de sus reuniones anuales ha sido de 3,9 desde su creación (Colino y Parrado, 2009: 151). Al mismo tiempo, resulta importante subrayar que este organismo multilateral ha sido muy efectivo en términos de número de acuerdos alcanzados, lo que también demuestra una práctica intergubernamental ampliamente asentada dentro del ámbito sanitario español.

Gráfico 1. Efectividad de Consejo Interterritorial del Sistema Nacional de Salud. Acuerdos/plenos (1987-2004)

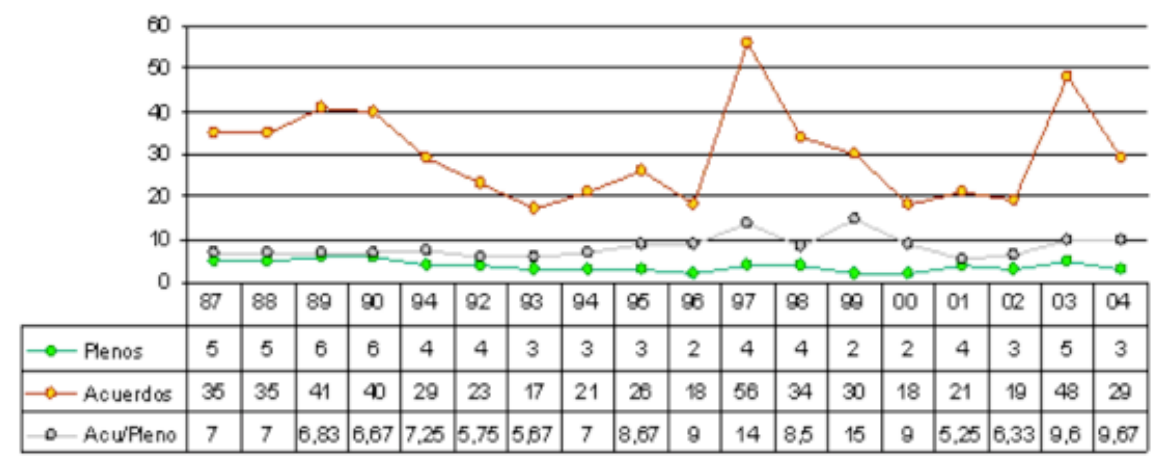

Fuente: Ministerio de Sanidad (2011).

Al mismo tiempo, se debe subrayar que una gran parte de los acuerdos alcanzados se refieren al régimen interior del propio órgano intergubernamental. Esto refleja la necesidad de llegar a acuerdos más allá del ámbito de la política pública concreta, configurándose una cooperación intergubernamental entre los miembros que componen el CISNS, que permite un estrechamiento de las relaciones, que favorece la posterior toma de decisiones. Así

En lo que respecta a las áreas temáticas a las que corresponden los asuntos tratados en los Plenos de estos cabe destacar que de los 542 acuerdos alcanzados, 202 (37\%) se refieren a asuntos de régimen interior (aprobación de actas, nombramientos, modificaciones de su reglamento, creación, disolución y modificación de ponencias, comisiones y grupos de trabajo) y el resto, 340 (63\%), constituyen propiamente los acuerdos y/o recomendaciones. (Ministerio de Sanidad, 2012).

Todo ello está unido a la Ley 16/2003, de 28 de mayo, de cohesión y calidad del Sistema Nacional de Salud, que redefinió los fundamentos de las interacciones entre las administraciones españolas con competencias en materia sanitaria. De hecho, dentro de la misma se prevé la constitución entre las diferentes administraciones de comisiones y comités técnicos, la celebración de convenios y la elaboración de programas en común que se requieran para la mayor eficacia y rentabilidad de los servicios sanitarios, al amparo del CISNS (Colino y Parrado, 2009). Entre las comisiones y grupos de trabajo del CISNS, cabe destacar algunos, tales como el Comité Técnico Conjunto Mínimo Básico de Datos, 
la Comisión Científico-Técnica del SNS, la Comisión Permanente de Trasplantes, la Comisión Permanente de Aseguramiento, Financiación y Prestaciones, la Comisión Permanente de Farmacia, la Comisión de Salud Pública o la Comisión de Seguimiento del Fondo de Cohesión Sanitaria (CISNS, 2009). Muchas de ellas cuentan a su vez con grupos de trabajo y ponencias donde se trabaja a un nivel más técnico.

Normativamente, la Ley 16/2003 (Capítulo I) encarga al Ministerio de Sanidad el tratamiento de las TIC y la implementación de los proyectos de acuerdo con el protocolo específico. En particular, esta normativa entiende a nivel general las tecnologías como servicios o medios para un fin, cuya implementación estará supervisada y controlada por el Ministerio de Sanidad a través de agencias de evaluación (art. 21). Consecuentemente, la tutela de las TIC y de los procedimientos tecnológicos en materia de salud depende de las administraciones sanitarias autonómicas y del Ministerio de Sanidad, previo acuerdo del CISNS.

De manera complementaria, esta normativa establece un apartado específico sobre la red de comunicaciones del Sistema Nacional de Salud. Dentro del art. 54, se reserva al Ministerio de Sanidad un papel esencial en la gestión de la mencionada red, manifestando que:

A través de la utilización preferente de las infraestructuras comunes de comunicaciones y servicios telemáticos de las Administraciones públicas, pondrá a disposición del Sistema Nacional de Salud una red segura de comunicaciones que facilite y dé garantías de protección al intercambio de información exclusivamente sanitaria entre sus integrantes.

La transmisión de la información en esta red estará fundamentada en los requerimientos de certificación electrónica, firma electrónica y cifrado, de acuerdo con la legislación vigente.

A través de dicha red circulará información relativa al código de identificación personal único, las redes de alerta y emergencia sanitaria, el intercambio de información clínica y registros sanitarios, la receta electrónica y la información necesaria para la gestión del Fondo de cohesión sanitaria, así como aquella otra derivada de las necesidades de información sanitaria en el Sistema Nacional de Salud.

Lo anterior se completa con el art. 56, en el que se plantea la necesidad de intercambio de información en salud entre organismos, centros y servicios del Sistema Nacional de Salud:

Con el fin de que los ciudadanos reciban la mejor atención sanitaria posible en cualquier centro o servicio del Sistema Nacional de Salud, el Ministerio de Sanidad y Consumo coordinará los mecanismos de intercambio electrónico de información clínica y de salud individual, previamente acordados con las comunidades autónomas, para permitir tanto al interesado como a los profesionales que participan en la asistencia sanitaria el acceso a la historia clínica en los términos estrictamente necesarios para garantizar la calidad de dicha asistencia y la confidencialidad e integridad de la información, cualquiera que fuese la Administración que la proporcione.

El Ministerio de Sanidad y Consumo establecerá un procedimiento que permita el intercambio telemático de la información que legalmente resulte exigible para el ejercicio de sus competencias por parte de las Administraciones públicas. 
En definitiva, las TIC se encuentran sujetas a una constante evaluación por el CISNS y se rigen por lo principios de eficiencia, eficacia y seguridad. La Ley 16/2003 considera las TIC como un servicio, un medio para mejorar la cooperación entre los diferentes agentes, garantizando la implementación de nuevos proyectos que permitan una modernización del sistema sanitario, mediante los mencionados principios de eficiencia, eficacia y seguridad. Todo lo anterior se plantea bajo la tutela y la evaluación de los órganos de gobierno principalmente CISNS y con la mirada puesta a la Unión Europea, que también promueve una renovación constante.

En el primer caso, la vertiente normativa dio pie a la creación de una Subcomisión de Sistemas de Información (SSI) en línea con otras comisiones y grupos de trabajo del CISNS, en este caso orientada a la promoción de la cooperación en materia tecnológica. La SSI tiene el objetivo de garantizar la cooperación entre diferentes actores del ámbito sanitario: autoridades administrativas, profesionales, ciudadanos, así como diferentes asociaciones y organizaciones. Por lo tanto, aquélla constituye una pieza fundamental para hacer frente a la compleja realidad organizativa de la política sanitaria española y de las demandas de los tres grandes grupos de actores que integran el sistema sanitario en materia tecnológica (Sistema de información Sanitaria del SNS, 2010:5): los ciudadanos, los profesionales del sector sanitario y las administraciones sanitarias mismas, las cuales cuentan con unos recursos limitados y perciben las TIC como una oportunidad de ofrecer el mejor servicio posible, con la máxima calidad de la manera más eficiente.

Dentro de la SSI se han ido abordando los diferentes aspectos intergubernamentales relacionados con las TIC e Internet dentro de la política sanitaria española. Sus actividades se inician en 2003, momento en que se acordó configurar su organización interna, delimitando funciones y actividades. Los proyectos concretos planteados en materia TIC se relacionaron con el desarrollo de la tarjeta sanitaria individual del SNS, el estudio de modelos de tarjetas, la atención a las bases de datos de usuarios, así como la firma de un real decreto sobre listas de espera (CISNS, 2003:175). En 2004 se creó un taller para desarrollar un proyecto sobre la gestión de las listas de espera, que se convirtió en objeto de preocupación de la SSI (CISNS, 2004:71). Durante el año 2005 se hizo una revisión de los proyectos aprobados en años anteriores, incluyendo el sistema de información de atención primaria, el sistema de registro de centros sanitarios y servicios o el convenio de impulso a la tarjeta sanitaria (CISNS, 2005:72).

El periodo que se inicia en 2006 implica un salto cualitativo en las actividades de la SSI. Dentro de la misma, se revisaron proyectos anteriores y se acordó la financiación de las acciones, a través de una dotación para que cada comunidad autónoma pudiera implantar los proyectos definidos (con una partida total de 2.962.750€ en 2006). También se produjo la adaptación de la información económica de la estadística de gasto sanitario público al marco conjunto EUROSTAT-OCDE-OMS, al mismo tiempo que se puso en marcha la Intranet Sanitaria para las nuevas aplicaciones diseñadas en servicios Web del SNS (CISNS, 2006:66). Durante el año 2007 se continuó en la misma línea que en 2006, dotando de financiación del SNS (un total de 3.962.750€) para repartir a las CCAA según criterios de población, insularidad, mínimo por comunidad, etc., en proyectos tecnológicos (CISNS, 2007:88). En el año de 2008 se hizo una evaluación sobre la situación de los datos de la actividad realizada hasta ese momento, presentando una base de datos de nuevos indicadores del SNS (CISNS, 2008:86). De la última memoria de funcionamiento del CISNS (año 2009) se desprenden las siguientes iniciativas promovidas por la SSI: 
proyecto REBECA (intercambio de datos e información estadística) y propuesta de ampliación a otros organismos, así como la promoción de la interoperabilidad de datos clínicos en el SNS, entre otros (CISNS, 2009).

En la siguiente sección se abordará de una manera más concreta el proyecto de Historia Clínica Electrónica, en el que el papel de la interoperabilidad ha llevado al liderazgo de la SSI. Lo importante aquí es poner el acento en que la interoperabilidad organizativa y su gobernanza han tenido en la SSI un actor esencial. A través de este grupo de trabajo de la CISNS se ha dotado de impulso a las iniciativas, así como de espacio de encuentro en el que tanto la administración central como las administraciones regionales han podido conversar sobre las iniciativas planteadas para mejorar la coordinación del SNS desde una vertiente tecnológica.

De manera complementaria, en esta vertiente organizativa y de gobernanza hay que subrayar que las actuaciones en materia de interoperabilidad en la política sanitaria se encuentran ligadas a las iniciativas de la Unión Europea dentro del Plan de Acción eHealth. Este marco de actuación aprobado en 2004 ha permitido a las administraciones públicas españolas desarrollar la interoperabilidad mediante el trabajo en red entre organizaciones, ampliando su ámbito de acceso a la información y posibilitando el intercambio de información dentro del sistema administrativo europeo (Comisión Europea, 2004). En concreto, el Plan de Acción eHealth convirtió la interoperabilidad en una de sus prioridades, sobre todo, en lo concerniente a la identificación de pacientes y las historias clínicas.

Recientemente, teniendo en cuenta la nueva estrategia Europe 2020 (Comisión Europea, $2010 b$ ), se ha abierto un proceso consultivo de cara a la elaboración de una nueva agenda sanitaria europea. De hecho, ya se ha aprobado un documento denominado Redesigning Health in Europe for 2020, elaborado por el EU Task Force on e-Health (Comisión Europea, 2012). Junto a lo anterior, se están desarrollado diferentes acciones y proyectos relacionados con la interoperabilidad en el ámbito sanitario. En concreto, hay varios proyectos que han desarrollado numerosas ideas sobre interoperabilidad dentro de esta política pública. A modo de ejemplo, se puede mencionar CALLIOPE (European thematic Network for eHealth Interoperability), epSOS (Smart Open Services for European Patients), HITCH (Healthcare Interoperability Testing and Conformance Harmonisation) o SemanticHealth, casos que se han convertido en referente para los estados miembros.

De manera particular, CALLIOPE es una experiencia específica en la que se ha puesto el acento en la necesidad de generar una red de actores especializados en la interoperabilidad en el ámbito sanitario. La red CALLIOPE se puso en marcha en $2008 \mathrm{y}$ fue creciendo en cuanto al número de actores presentes dentro de la misma, incluyendo administraciones públicas, asociaciones, empresas del sector, etc. Dentro de ella se ha potenciado un diálogo sobre interoperabilidad sanitaria a nivel europeo. Además, se ha validado una metodología de trabajo que permite una interacción y apertura considerable dentro de la materia entre la comunidad especializada en la sanidad en Internet. Por su parte, epSOS es otro proyecto en el que la interoperabilidad sanitaria tiene un papel centro y se ha convertido en un impulsor de la receta electrónica y la historia clínica electrónica de los pacientes.

En suma, el papel de la UE en este ámbito es orientador y en ningún caso los estados miembros y sus diferentes niveles administrativos se encuentran comprometidos por las iniciativas adoptadas o los proyectos que se llevan a cabo en ese nivel supranacional. A pesar de que la política sanitaria es una de las que ha planteado proyectos de 
interoperabilidad más evolucionados (Criado, 2012), las instituciones comunitarias no tienen un papel definitivo a nivel interno de los estados. No obstante, en el caso español se ha contemplado a las instituciones comunitarias como un referente que establece prioridades, define objetivos, ofrece recursos, difunde iniciativas y/o genera conocimiento. Por consiguiente, también es necesario tener en cuenta ese rol referencial, simbólico y de legitimación desempeñado por las instituciones comunitarias.

\subsection{Aspectos técnico-semánticos}

Desde un punto de vista técnico-semántico, a partir del año 2003 también entraron en funcionamiento servicios Web del Sistema Nacional de Salud (SNS). Estos servicios son los que fundamentan el intercambio de información para diferentes aspectos comunes, tales como la Base de Datos de Usuarios de Tarjeta Sanitaria, el Fondo de Cohesión y, progresivamente, otros que se puedan consolidar en el futuro. Los servicios del SNS son los que hacen efectiva la interoperabilidad en el ámbito sanitario con la integración de los sistemas de las administraciones autonómicas. Para ello se usan estándares de intercambio de datos a través de XML, lo que facilita la independencia de las plataformas y las aplicaciones. En otras palabras, sean cuales sean los sistemas que se utilizan en las administraciones implicadas se garantiza su interconexión a estos servicios comunes.

El Ministerio de Sanidad establece algunas características de lo que ha venido a llamar esquema de interoperabilidad en el ámbito sanitario (Ministerio de Sanidad, 2011).

Figura 2. Servidor de intercambios del Sistema Nacional de Salud

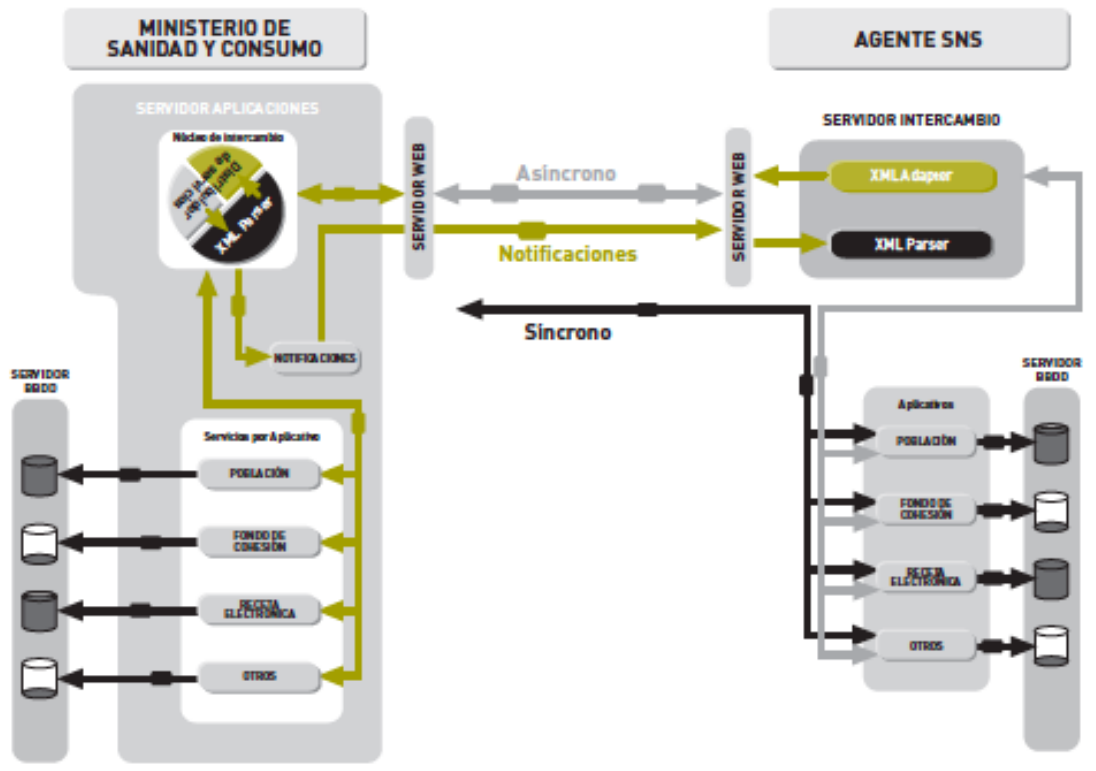

Fuente: Ministerio de Sanidad (2011).

En primer lugar, la vertiente del núcleo central de servicios pone de relieve que el servidor del SNS está compuesto por ese núcleo para el tratamiento de mensajes y servicios específicos proporcionando la funcionalidad necesaria. El núcleo del SNS es un 
servidor centralizado, accesible a través de una intranet sanitaria, que permite el acceso al SNS a través de una red exclusiva y dedicada (ver Figura 1).

El acceso de los sistemas cliente al SNS a través de esa red ofrece la garantía en cuanto a los niveles de servicio, el ancho de banda, el tiempo de respuesta y el incremento de la seguridad del sistema.

Por otro lado, el SNS no cuenta con usuarios físicos registrados en el sistema. Lo anterior significa que el intercambio de información se realiza siempre entre los servidores de los sistemas agente y el servidor central. La autentificación de los sistemas cliente (servicios de salud) se realiza mediante la utilización de certificados digitales X509v3. Éstos identifican a cada uno de los servidores que acceden al sistema y al servidor propio del SNS. Las aplicaciones instaladas oficialmente en los servidores de los servicios autonómicos de salud son las únicas responsables de generar el correspondiente mensaje XML.

En tercer lugar, la garantía de las comunicaciones entre los sistemas de cliente y el núcleo del SNS se logra con la encriptación mediante el protocolo SSL v3. Con ello se pretende una observancia completa de la privacidad de la información y la exposición de la misma a terceros no autorizados. De igual forma, todos los mensajes de intercambio entre el SNS y los sistemas de cliente van firmados digitalmente por el emisor y comprobados por el receptor. De esta manera, se logra una garantía de la integridad de los datos e información y del no repudio.

Otro aspecto clave desde el plano técnico se refiere a la independencia de las plataformas. La base de datos del SNS puede operar con cualquier sistema que sea capaz de generar, enviar, recibir y procesar mensajes XML. Al ser XML el estándar de intercambio, el sistema se encuentra preparado para una rápida integración con otras aplicaciones o sistemas que utilicen dicho estándar, no sólo en el ámbito nacional, sino europeo o mundial.

En quinto lugar, en cuanto a la integración de nuevos servicios, se realiza mediante la definición de nuevos mensajes XML, facilitando la prestación de nuevas funcionalidades a través de la reutilización de la plataforma existente. La definición de nuevos servicios en el SNS implica el desarrollo de los módulos de tratamiento de la información en los sistemas cliente: la generación del XML a partir de la información contenida en los sistemas y el tratamiento de los mensajes recibidos. Todo el núcleo de intercambio, la gestión de colas y mensajes y los procedimientos de localización y de seguridad forman parte del núcleo del SNS, no siendo necesario implementar elementos adicionales en los nuevos servicios.

Finalmente, queda la vertiente de la implementación de procedimientos de calidad, que se pueden establecer desde el nodo central. A modo de ejemplo, en el caso especial del servicio de tarjeta sanitaria, el SNS genera un código único de identificación para cada persona protegida, que es accesible desde todas las comunidades autónomas, posibilitando con ello la interoperabilidad de las diferentes tarjetas, al mismo tiempo que se mantienen los elementos particulares de cada una de ellas.

\section{ESTUDIO DE CASO. LA HISTORIA CLÍNICA DIGITAL DEL SISTEMA NACIONAL DE SALUD}

\subsection{Descripción general del caso}

El Ministerio de Sanidad de España ha trabajado desde el año 2006 para desarrollar un sistema común para el SNS que permita la interoperabilidad de los datos sanitarios de los ciudadanos que puedan existir en varias comunidades autónomas. Este proyecto se ha 
definido con el nombre de Historia Clínica Digital del SNS (HCDSNS), que tiene como fin básico posibilitar el acceso controlado a la información de un paciente desde cualquier punto asistencial del SNS, primario o especializado, sin interferir en los sistemas propios de los servicios de salud y contando como único fin el estrictamente asistencial, siempre que así lo solicite el paciente (CISNS, 2008). La visión ha consistido en la normalización de historias clínicas para el SNS y, especialmente, establecer el mínimo de datos que son requeridos para lograr la interoperabilidad en el conjunto del sistema sanitario, así como la heterogeneidad de los contenidos ofrecidos a pacientes y profesionales. A día de hoy todavía no se ha cerrado completamente el proyecto, en parte como consecuencia de las dificultades de poner de acuerdo a todos los actores del sistema, a pesar de que la mayoría de elementos semánticos, técnicos, e incluso organizativos, de la interoperabilidad han quedado resueltos. Por consiguiente, la vertiente de la gobernanza de la interoperabilidad es un factor clave a la hora de caracterizar el proceso de implantación de un proyecto de interoperabilidad sanitaria de este nivel de complejidad.

El punto de partida del proyecto se posiciona en la propia naturaleza descentralizada del modelo sanitario español, en el que la competencia de gestión de la asistencia sanitaria pública se encuentra en manos de los servicios de salud autonómicos. Lo anterior supone la imperiosa necesidad de instrumentos adecuados de coordinación y cohesión, por lo que la historia clínica se convierte en un ejemplo de gran interés. A mediados de la década anterior todas las administraciones sanitarias autonómicas españolas había logrado ya casi una total implantación de la Historia Clínica Electrónica (HCE). Sin embargo, el acceso a esta información viene quedando restringido al ámbito de la administración sanitaria autonómica correspondiente, por lo que cuando el paciente requiere atención fuera de sus límites geográficos la información no está accesible para los profesionales del SNS ni para los propios ciudadanos. Por ello, en 2006 se inicia el proyecto HCDSNS.

Los objetivos generales planteados en el origen del proyecto fueron los siguientes (Agencia de Calidad del SNS, 2010:10):

* Garantizar al ciudadano el acceso por vía telemática a los datos de salud, propios o de sus representados, que se encuentren disponibles en formato digital en alguno de los Servicios de Salud que se integran en el SNS, siempre que cumplan los mínimos requisitos de seguridad establecidos para proteger sus propios datos contra la intrusión ilegítima de quienes no hayan sido facultados para acceder.

* Garantizar a los profesionales sanitarios, facultados por cada Servicio de Salud para esta función y autorizados en cada caso por el paciente, el acceso a determinados conjuntos de datos de salud, generados en una Comunidad Autónoma distinta de aquélla desde la que se requiere la información, siempre que el usuario o paciente demande sus servicios profesionales desde un centro sanitario público del SNS.

* Dotar al SNS de un sistema seguro de acceso que garantice al ciudadano la confidencialidad de los datos de carácter personal relativos a su salud.

* El sistema a desarrollar deberá dotarse de agilidad y sencillez en el acceso, al servicio de ciudadanos y profesionales.

La financiación del proyecto de HCDSNS se ha concretado a través de diferentes vías. Desde su origen se han definido tres vías: el programa Sanidad en Línea de la Administración General del Estado (administración central), los Fondos de Cohesión destinados a financiar Estrategias de Salud, de acuerdo con lo previsto en la Ley 16/2003 de 28 de mayo de Cohesión y Calidad del SNS (administración europea) y la firma de convenios específicos para cofinanciar las actuaciones preliminares del piloto junto a los 
gobiernos autonómicos participantes (administración central y administraciones regionales).

Finalmente, cabe subrayar en esta presentación general que la orientación del proyecto no lleva a que se considere el acceso a la totalidad de la HCE, sino sólo compartir conjuntos de datos clínicos considerados relevantes. En otras palabras, se propuso un diseño funcional del sistema que permitiera el acceso de los profesionales y ciudadanos a aquellos datos que son relevantes desde el punto de vista clínico, dado que resumen lo esencial del contenido del historial clínico del ciudadano. Se pasa a continuación a ver aspectos más concretos del caso relacionados con los diferentes tipos de interoperabilidad apuntados más arriba.

\subsection{Interoperabilidad organizativa}

En este apartado se plantean aquellos aspectos que tienen que ver con la definición de los procesos de las organizaciones implicadas en el despliegue de la HCDSNS. De entrada, la HCDSNS tiene cobertura normativa en la mencionada Ley 16/2003. En su artículo 56, la Ley 16/2003 dirigió al Ministerio de Sanidad el mandato de coordinar los mecanismos de intercambio electrónico de información clínica y salud individual, para permitir el acceso, tanto al usuario como a los profesionales, en los términos estrictamente necesarios para garantizar la calidad de la asistencia y la confidencialidad e integridad de la información. A pesar de ello, tanto la administración central, como las administraciones autonómicas se plantearon la necesidad de articular una solución concertada para llevar a cabo las actuaciones ligadas a la HCDSNS.

Al mismo tiempo, una normativa posterior ha establecido un hito clave para la entrada en vigor del proyecto. Así, el Real Decreto Ley 9/2011, de 19 de agosto, de medidas para la mejora de la calidad y cohesión del sistema nacional de salud, de contribución a la consolidación fiscal, y de elevación del importe máximo de los avales del Estado para 2011, establece en su artículo 6 lo siguiente:

De acuerdo con lo expresado en el artículo 56 de la Ley de Cohesión y Calidad del Sistema Nacional de Salud y su desarrollo reglamentario en relación al conjunto mínimo de datos de los informes clínicos del Sistema Nacional de Salud y a los efectos de hacer efectivo tanto al interesado como a los profesionales que participan en la asistencia, un acceso adecuado a la historia clínica en todo el Sistema Nacional de Salud en los términos previstos por el ordenamiento jurídico, las administraciones sanitarias establecerán de manera generalizada la conexión e intercambio de información con el Sistema de Historia Clínica Digital del SNS, antes del 1 de enero de 2013 .

En consecuencia, a partir de esta fecha debe estar en funcionamiento todo el sistema.

Junto a lo anterior, otro de los fundamentos normativos de la HCDSNS se refiere al Decreto 1093/2010, de 3 de septiembre, por el que se aprueba el conjunto mínimo de datos de los informes clínicos en el Sistema Nacional de Salud. Dentro del mismo se definieron los documentos electrónicos que contienen los datos auténticamente relevantes como soporte de información para la atención sanitaria de un ciudadano que demanda asistencia: información de atención primaria; informe clínico de urgencias; informe clínico de alta; informe clínico de consulta externa de especialidades; informe de cuidados de enfermería; informe de resultados de pruebas de laboratorio; informe de resultados de pruebas de imagen; informe de resultados de otras pruebas diagnósticas. Cada uno de estos documentos electrónicos se ubican en las historias clínicas de los pacientes, independientemente de las administraciones sanitarias autonómicas. De manera complementaria, se ha creado la historia clínica resumida, 
conjunto de datos creado ad hoc y destinado a ser usado por los profesionales sanitarios.

Esta última normativa se puede considerar un punto de llegada esencial en el camino de la normalización de los procesos necesarios para hacer viable la HCDSNS. De hecho, establece unos requerimientos que deben ser cumplidos por el conjunto de las administraciones públicas involucradas. Esto es, se trata de legislación básica que las administraciones autonómicas deben seguir en el ejercicio de sus competencias sanitarias, fundamentada en lo establecido en el artículo 56 de la Ley 16/2003, de 28 de mayo. En consecuencia, desde un punto de vista organizativo, esta normativa establece los aspectos básicos del procedimiento a desarrollar, en la medida que delimitan los rasgos de los documentos clínicos que conforman la HCDSNS.

\subsection{Interoperabilidad técnica}

Desde un punto de vista técnico, el proyecto de HCDSNS ofrece unas lecciones de gran interés para otras iniciativas similares dentro del sector sanitario. En primera lugar, hay que indicar que la interoperabilidad técnica de la HCDSNS se ha fundamentado en una estrategia basada en la neutralidad tecnológica, a fin de no condicionar las decisiones de las comunidades autónomas apostando por la adopción de estándares abiertos, tal y como se definen en el Marco Europeo de Interoperabilidad (2004), así como todas las iniciativas de interoperabilidad de la Unión Europea (Criado, 2012). Lo anterior implica establecer una capa que permita la transmisión de datos de los distintos sistemas que los actores que forman parte del SNS puedan implantar sin que ello condicione, no sólo las soluciones adoptadas por cada una de las administraciones sanitarias regionales, sino también todas aquellas que decidan adoptarse en el futuro por cada una de ellas, teniendo en cuenta que disponen de autonomía a la hora de ejercer sus competencias.

Por otro lado, hay que subrayar la existencia de un grupo de trabajo de Estándares y Requerimientos Técnicos (GERT) para la HCDSNS, que ha desarrollado una política de estándares que integra una serie de recomendaciones sobre los siguientes aspectos (Agencia de Calidad del SNS, 2010: 24):

* El formato de intercambio de datos (XML)

* Formatos de documentos a intercambiar (PDF)

* Formato de imagen (DICOM)

* Tablas de codificación del Instituto Nacional de Estadística para los códigos de CCAA, provincias, municipios, etc.

* Identificación de pacientes de forma unívoca mediante el Código de Identificación Personal asociado al código de las comunidades autónomas

* Identificación de profesionales (DNI/NIE/Pasaporte) o número de colegiado

* Formato del NIE

* Certificados de seguridad

* Intercambio de información clínica (HL7 CDA nivel 1 para cabeceras de documentos).

Desde un punto de vista técnico, el sistema que soporta la HCDSNS es el mencionado Nodo Central de Intercambio del Ministerio de Sanidad, así como los estándares tecnológicos planteados. El Nodo Central del SNS es la infraestructura hardware y software que facilita intercambio de información (administrativa y clínica) entre los distintos agentes del SNS: administraciones autonómicas, mutualidades sanitarias, así como otros organismos competentes en la materia sanitaria. El hecho de implantar en el Nodo Central de Intercambio del SNS una arquitectura orientada a servicios Web (SOA), tal y como se ha manifestado más arriba, ha hecho posible incorporar éstos de forma 
progresiva sin que sea necesario unificar las aplicaciones existentes en las administraciones autonómicas, modificar el software de base, ni establecer integraciones a medida entre los sistemas de los distintos agentes. Todo lo anterior se ha realizado con un fundamento concreto en la Propuesta técnica para la puesta en marcha de un piloto para el intercambio de datos de la HCDSNS, basado en los servicios Web del SNS (GERT, 2008). Este documento explicita de una manera más concreta todos los detalles técnicos del proyecto.

Desde el punto de vista de la seguridad, se ha planteado que este sistema tiene un valor estratégico, dada su criticidad y la naturaleza de los datos de carácter personal que requieren el más alto nivel de protección de los que establece la Ley Orgánica 15/1999, de 13 de diciembre, de Protección de Datos de Carácter Personal (así como el Real Decreto 1720/2007, que aprueba su reglamento de desarrollo). En particular, se plantea la necesidad de reforzar las medidas de control previo para el acceso mediante el uso de certificación electrónica, dado que ofrece mayores garantías de autenticidad, la asignación de los profesionales a grupos distintos según la función que desempeñen con acceso a contenidos de información diferenciados según el grupo (Ministerio de Sanidad, 2012). Al mismo tiempo, la seguridad se refuerza, sobre todo, con sistemas de control posterior mediante acceso de los propios ciudadanos a los registros de auditoría interna de la HCDSNS. En lo anterior juega un papel relevante el propio ciudadano, como auditor externo, al poder llevar a cabo el seguimiento de los accesos realizados a sus datos de salud.

\subsection{Interoperabilidad semántica}

Como ya se ha explicitado más arriba, la interoperabilidad semántica resulta esencial para llevar a cabo un proyecto complejo de e-Administración. En particular, hace posible el intercambio de datos, facilita la interpretación de su significado, su integración en los sistemas de destino con su información de contexto. En otras palabras, permite construir y transmitir conocimiento. Al mismo tiempo, opera para que los lenguajes encaminados a representar el contenido de los documentos sean identificados e interpretados del mismo modo por usuarios que utilizan diferentes clasificaciones (o idiomas). Teniendo en cuenta lo anterior, el Grupo asesor de interoperabilidad semántica de la HCDSNS (GAISHC) se planteó proponer recomendaciones orientadoras de las líneas de acción en este campo, de cara a resolver el problema de la interoperabilidad semántica de la HCDSNS.

Así, se han planteado una serie de necesidades comunes entre los distintos agentes que participan en el intercambio de información. Por ejemplo, disponer de un vocabulario único normalizado que sea reconocido por todos los sistemas implicados. Junto a los vocabularios, el GAISHC estableció la necesidad de disponer de una serie de instrumentos que facilitan la interoperabilidad semántica: los arquetipos, las plantillas y un modelo de referencia (GAISHC, 2008).

De entrada, se decidió iniciar una línea de trabajo orientada a poner a disposición del conjunto de los agentes del SNS los elementos semánticos precisos para alcanzar la interoperabilidad de la HCDSNS. Así, se adoptó un vocabulario sanitario controlado y estandarizado para lograr la interpretación inequívoca y automática de los contenidos transmitidos entre sistemas distintos de forma precisa y en idiomas diferentes, facilitando el acceso a la información relevante para la toma de decisiones clínicas. Se eligió Snomed Clinical Terms (Snomed-CT) como terminología clínica de referencia internacional, que goza de un mayor reconocimiento y uso más extendido. 
Tal y como manifiesta el Ministerio de Sanidad en su portal web (2012), el Snomed CT es un producto que nace de la fusión entre Snomed RT (Snomed Reference Terminology), creada por el College of American Pathologists y el Clinical Terms Version 3 (CTV3), desarrollada por el National Health Service (NHS) del Reino Unido. Esta fusión ha permitido la combinación de los términos en los ámbitos de las ciencias básicas, la bioquímica y las especialidades médicas de Snomed RT, con los contenidos de la atención primaria del CTV3. Todo lo anterior ha dado lugar a una terminología de referencia que permite a los profesionales de la salud de todo el mundo representar la información clínica de forma precisa e inequívoca, en formato multilingüe. Actualmente, esta terminología es mantenida y distribuida por la International Health Terminology Standards Development Organisation (IHTSDO), de manera que fue necesario el ingreso del SNS en la dicha organización, como requisito previo para el uso de Snomed CT.

En definitiva, lo anterior permite considerar que la interoperabilidad semántica en el ámbito sanitario está en línea con los estándares internacionales, lo que ha facilitado también el despliegue de la HCDSNS (Agencia de Calidad del SNS, 2010: 29-30). Este caso también se fundamenta en la existencia de unos arquetipos comunes, como modelos de datos orientados a representar una manifestación de la realidad, formando definiciones de conceptos clínicos (informe de alta, análisis de glucosa, historia familiar, etc.). Por su parte, también se usan plantillas, esto es, una serie de restricciones adicionales en un modelo estático normalizado, que sirven para representar un nivel deseado de estructura y contenido de la información con el propósito de transmitirlo de una forma ordenada y explícita. Finalmente, se planteó la necesidad de establecer un modelo de referencia para llevar adelante el proyecto de HCDSNS desde la óptica de la interoperabilidad semántica. En particular, se planteó la conveniencia de asumir un modelo dual, como el estándar europeo ISO/CEN EN13606, en el que los datos y el conocimiento se representan de una manera independiente, si bien muy relacionados.

\subsection{Gobernanza de la interoperabilidad}

Desde el plano de la gobernanza de la interoperabilidad, la complejidad está ligada a la variedad de actores y grupos que participan de un proyecto tan complejo como es el de la HCDSNS. En general, la HCDSNS ha tenido una cierta conciencia de la necesidad de concertar con diferentes actores relacionados con las diferentes vertientes del mismo. De hecho, la ejecución de las diferentes tareas a desarrollar en los diferentes momentos del proceso se fundamentó en dos etapas (Agencia de Calidad del SNS, 2010:20): una fase de consenso profesional y una fase de consenso institucional (ver Figura 3).

La fase de consenso profesional implicó la participación de los profesionales sanitarios en la definición del proyecto. Según lo anterior, se constituyeron grupos de trabajo compuestos por profesionales del ámbito de la salud, cuyos perfiles de experiencia fueron descritos en función de los contenidos a definir, integrando también a la ciudadanía en la caracterización de las funcionalidades del sistema (Agencia de Calidad del SNS, 2010). Después de la definición de los perfiles, se elevaron a las diferentes sociedades científicas y ciudadanas, de cara a nombrar a las personas adecuadas para su desempeño. Así, treinta $\mathrm{y}$ dos sociedades científicas $\mathrm{y}$ asociaciones civiles nombraron cuarenta $\mathrm{y}$ cinco representantes, mientras que el Ministerio de Sanidad designó a quince profesionales expertos en admisión, documentación clínica y gestión de centros, con el fin último de crear siete grupos de trabajo. 
Figura 3. Evolución del plan de trabajo del proyecto HCDSNS

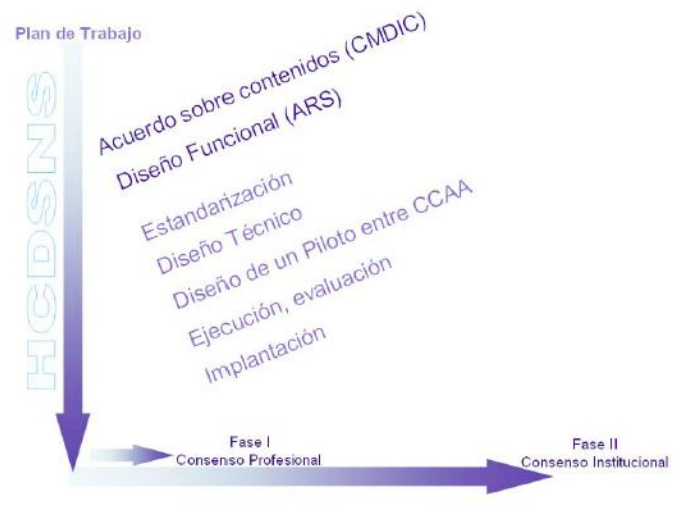

Fuente: Agencia de Calidad del SNS (2010:20).

La manera de operar de estos grupos de trabajo fue virtual, bajo la dirección del Ministerio de Sanidad, de manera que los trabajos se fueron concretando vía web. En concreto, Se logró un consenso respecto del conjunto mínimo de datos de informes clínicos requeridos para la HCDSNS (Ministerio de Sanidad, 2008a). A la vez, se llegó a un acuerdo sobre los requerimientos de carácter funcional y de seguridad del sistema, que quedaron recogidos dentro del documento Análisis de Requerimientos del Sistema (Ministerio de Sanidad, 2008b). Como se puede observar, estos primeros trabajos se desarrollaron a un nivel de carácter técnico, lo que facilitó de una manera importante su ejecución.

La fase de consenso institucional implica una vertiente más compleja de la gobernanza de la interoperabilidad, en la medida que las implicaciones políticas son más notorias. En un primer momento, se llevó a cabo una validación de los primeros consensos profesionales por parte de todos los responsables de la política sanitaria en cada uno de los diecisiete gobiernos autonómicos. Las conclusiones iniciales de los expertos autonómicos en el proyecto HCDSNS fueron elevadas a la SSI y, finalmente, al CISNS. De este modo, este máximo órgano de gobernanza de la política sanitaria en España acordó en su reunión de 10 de octubre de 2007 aprobar el diseño acordado inicialmente. Junto a lo anterior, se crearon nuevos grupos de trabajo orientados al despliegue completo de la interoperabilidad técnica, semántica, así como las condiciones para la realización del piloto del proyecto (Agencia de Calidad del SNS, 2010).

El Grupo de trabajo de estándares y requerimientos técnicos de la HCDSNS (GERT) se ha compuesto por expertos con perfil tecnológico de cada administración autonómica y se ha encargado de revisar el diseño técnico del sistema de HCDSNS, definido por el Ministerio de Sanidad, consensuando una política de estándares técnicos para el SNS de cara al futuro, en línea con lo planteado más arriba.

El Grupo asesor de interoperabilidad semántica de la HCDSNS (GAISHC) ha tenido por objetivo proponer recomendaciones para orientar las líneas de acción en este campo, de cara a resolver el problema de la interoperabilidad semántica de la HCDSNS, incluyendo en ella el soporte a la diversidad lingüística. Se han priorizado aquellas soluciones necesarias para garantizar la interoperabilidad de una Historia Clínica 
Resumida. Sus miembros, designados por el Ministerio de Sanidad, son técnicos del SNS con experiencia concreta en este campo.

El Grupo de Comunidades para el pilotaje de HCDSNS (GCPHC) integra las diez administraciones autonómicas que han manifestado su decisión de participar activamente en el pilotaje de este sistema en escenarios reales. Las restantes administraciones sanitarias que decidieron actuar como observadoras del proceso de piloto del proyecto, también han dispuesto de la información actualizada de la evolución y resultado de los trabajos realizados, a través del espacio de trabajo, y se han incorporado a reuniones presenciales.

Estos grupos de trabajo han sido el soporte de la gobernanza del sistema, de manera que las decisiones han sido aprobadas por el mencionado SSI del CISNS. Ese aspecto ha supuesto un cierto retraso en el proceso de implantación de los consensos logrados a un nivel más técnico. De hecho, las reuniones de la SSI son limitadas a lo largo del año, de manera que el proceso de implantación del proyecto de HCDSNS se ha ralentizado de una manera importante, en la medida que cada uno de los avances planteados ha debido aprobarse a posteriori por la instancia política mencionada (CISNS), en la que los acuerdos son más difíciles de lograr.

Finalmente, y desde el punto de vista financiero, ya se ha mencionado que el proyecto HCDSNS se ha nutrido de tres ámbitos: el programa Sanidad en Línea, los Fondos de Cohesión y la firma de convenios específicos para cofinanciar las actuaciones preliminares del piloto junto a los gobiernos autonómicos participantes. En consecuencia, la financiación de este proyecto ha estado liderada por la Administración General del Estado, dentro de los planes AVANZA, de manera que las aportaciones realizadas por las administraciones autonómicas han sido mucho más modestas. A modo de ejemplo, en la tercera de las líneas planteadas en el año 2008, el Ministerio de Sanidad propuso la firma de un Convenio con cada una de las comunidades autónomas participantes en la primera fase del estudio piloto, cuyo objetivo era dar soporte a las acciones para iniciar la experiencia de intercambio real. Cada comunidad autónoma contribuía con $25.000 €$ al desarrollo de las acciones y el Ministerio de Sanidad con 200.000€. El Convenio se formalizó con siete gobiernos regionales, si bien a su vencimiento sólo cinco de las comunidades autónomas cumplieron los requerimientos contenidos en el mismo.

\section{DISCUSIÓN Y CONCLUSIONES}

La presente sección pretende abrir una discusión y ofrecer una serie de conclusiones ligadas a la interoperabilidad de e-Administración teniendo en cuenta el sector público español. Estas reflexiones finales se derivan del análisis realizado en el caso planteado, por lo que tienen una orientación hacia la dimensión digital de la política sanitaria. Al mismo tiempo, algunas de las conclusiones planteadas también tienen la voluntad de ofrecer una visión de mayor alcance sobre los proyectos de e-Administración. En consecuencia, se espera que puedan ayudar a entender las dinámicas que están detrás de las iniciativas de interoperabilidad que ya se llevan a cabo dentro de las administraciones públicas españolas.

Uno de los primeros aspectos a mencionar es que la política sanitaria española es un ejemplo paradigmático de las necesidades de colaboración intergubernamental en materia tecnológica en estados altamente descentralizados. En particular, el hecho de que las administraciones regionales españolas gestionen la sanidad en cada uno de sus ámbitos 
geográficos ha supuesto un reto para todo el Sistema Nacional de Salud. En ese sentido, los diferentes actores que participan en el sistema son conscientes de que la calidad de la prestación del servicio depende, en no poca medida, de la capacidad de llegar a acuerdos entre todos ellos. En el caso español, la política sanitaria ha gozado de unos niveles aceptables de acuerdo, de manera que las diecisiete administraciones autonómicas han actuado de manera concertada ante retos comunes junto a la Administración central, así como otros actores importantes del sector (ciudadanos, sociedades médicas, proveedores de servicios, mutualidades, etc.).

Teniendo en cuenta lo anterior, esta investigación ha puesto de manifiesto que las TIC se han convertido en un aliado ideal para llevar a cabo acciones que facilitan la concertación de los diferentes actores implicados en la política sanitaria. La interoperabilidad dentro del ámbito sanitario español se ha ido consolidando de varias maneras. Una de ellas es la existencia de un órgano administrativo intergubernamental que facilita el encuentro y acuerdo entre las diferentes administraciones públicas implicadas (Subcomisión de Sistemas de Información, dependiente del Consejo Interterritorial del Sistema Nacional de Salud). Dentro de la misma, se ha operado una aproximación de los actores técnicos del sistema, lo cual ha impulsado numerosas acciones.

La necesidad de un acompañamiento de naturaleza política, se pone todavía más de manifiesto en el ámbito del caso concreto analizado en este trabajo: la Historia Clínica Digital del Sistema Nacional de Salud (HCDSNS). El análisis del caso ha puesto de manifiesto el alto grado de consenso a la hora de definir la vertiente técnica y semántica de la interoperabilidad del sistema. De la misma manera, se puede considerar un éxito que la normativa de soporte para los nuevos procesos administrativos ligados a la HCDSNS se haya aprobado en un espacio de tiempo relativamente corto (cinco años). Al mismo tiempo, la gobernanza de la interoperabilidad del proyecto ha funcionado de una manera satisfactoria, si bien es cierto que la implantación definitiva de la interconexión entre las administraciones participantes no se ha completado. En otras palabras, el impulso político necesario para llevar a cabo la interoperabilidad no se ha concretado de una forma completa.

Desde una perspectiva más general, lo anterior es complementario con la necesidad de fomentar una cultura de la colaboración intergubernamental dentro del sector público, en todos sus niveles, y entre todos los empleados públicos. Del lado del capital humano resulta imprescindible la existencia empleados públicos con la capacitación requerida de cara a desarrollar proyectos de e-Administración con participación de agencias diferentes (Criado, et al., 2011). Así, el fortalecimiento de una cultura administrativa orientada a la colaboración intergubernamental debe cimentarse sobre un liderazgo híbrido (habilitado para gestionar tanto redes de políticas públicas como redes tecnológicas), que sea capaz de traducir las aspiraciones políticas en actuaciones configuradoras de servicios públicos digitales interoperables y de valor añadido para la ciudadanía.

Complementariamente, es preciso tener en cuenta también a los ciudadanos como receptores de las actuaciones en materia de interoperabilidad. Este aspecto implica identificar áreas de trabajo con valor añadido para ellos, teniendo en cuenta también las propias necesidades de las administraciones públicas, así como evaluar las iniciativas implantadas con un espíritu de mejora continua. Las administraciones públicas españolas ya se encuentran desarrollando proyectos de interoperabilidad en diferentes áreas clave, singularmente, compras públicas, tributos o seguridad social, mostrando una clara aproximación a las necesidades de la ciudadanía. Pero esas actuaciones se pueden extender 
a ámbitos como la salud, la justicia o la educación que conforman áreas claves para eliminar las desigualdades en el uso de las TIC.

En términos más académicos y relacionados con el sector público, las iniciativas de interoperabilidad deben evaluarse en función de las innovaciones, arreglos o implementaciones a las que den lugar. Además, dentro de esa valoración también hay que incluir la realización y grado de éxito de éstas y la satisfacción tanto de los actores involucrados como de los destinatarios finales (especialmente ciudadanos pero, también, organizaciones y empresas), de cara a lograr el objetivo de conseguir la sucesiva mejora de los componentes de los proyectos puestos en marcha. De la misma manera que se ha ilustrado a través de este trabajo, la evaluación es una herramienta de análisis que permite gestionar y dirigir adecuadamente las actuaciones en materia de interoperabilidad y obtener enseñanzas y experiencias para futuros proyectos. Este artículo espera haber contribuido en esa tarea, cada vez más importante en un área de expansión creciente como lo es la eAdministración.

\section{AGRADECIMIENTOS}

Este artículo se ha realizado con el apoyo del proyecto Barreras al gobierno electrónico. El empleo de las TIC en la política sanitaria autonómica. Un enfoque desde las relaciones intergubernamentales, financiado por el Ministerio de Economía y Competitividad de España (CSO2009-09169). Una versión previa de este trabajo se presentó en el XVII Congreso del CLAD, Cartagena de Indias, Colombia, 30 de octubre-2 de noviembre de 2012, dentro del panel La gestión de la interoperabilidad en Gobierno Electrónico en las administraciones públicas de Iberoamérica. Se agradece a todos los asistentes al panel sus valiosas preguntas, que ayudaron a mejorar este trabajo.

\section{BIBLIOGRAFÍA}

CISNS. (2009), Memoria de Actividades. Madrid, CISNS.

CISNS. (2008), Memoria de Actividades. Madrid, CISNS.

CISNS. (2007), Memoria de Actividades. Madrid, CISNS.

CISNS. (2006), Memoria de Actividades. Madrid, CISNS.

COLINO, C y PARRADO, S. (2009), 'La Institucionalización de los Instrumentos de Relación entre Estado y CCAA', en X. Arbós, (ed.) Las Relaciones Intergubernamentales en el Estado Autonómico. La posición de los actores. Barcelona, Institut d' Estudis Autonòmics.

COMISIÓN EUROPEA. (2004), eHealth Action Plan. Bruselas, Comisión Europea.

COMISIÓN EUROPEA. (2010a), A Digital Agenda for Europe, Bruselas, Comisión Europea.

COMISIÓN EUROPEA. (2010b), The European eGovernment Action Plan 2011-2015. Harnessing ICT to Promote Smart, Sustainable \& Innovative Government. Bruselas, Comisión Europea.

COMISIÓN EUROPEA. (2010c), European Interoperability Framework for European Public Services $(E I F)$. Bruselas, Comisión Europea, en: http://ec.europa.eu/isa/strategy/doc/annex_ii_eif_en.pdf.

CRIADO, J. I. (2009), Entre Sueños Utópicos y Visiones Pesimistas. Internet y las TIC en la Modernización de las Administraciones Públicas, Madrid, Instituto Nacional Administración Pública.

CRIADO, J. I. y NAVARRO, C. (2010): “Treinta años de políticas de modernización administrativa en España. Entre la inercia burocrática y la innovación tecnológica en la Administración General del Estado (1978-2008)", en: Revista Vasca de Administración Pública 86, pp. 61-99. 
CRIADO, J. I., GASCÓ, M. y JIMÉNEZ, C.E. (2011), 'Interoperabilidad del Gobierno electrónico en Iberoamérica. Estudio y Recomendaciones de Futuro'. Reforma y Democracia 50, pp.75-104.

DOS SANTOS, E (2007), "Implementing Interoperability Standards for Electronic Government: An Exploratory Case Study of the E-PING Brazilian Framework", International Journal of Electronic Government Research, 4(3), pp.103-112.

GAMERO, E. (2009), 'Interoperabilidad y Administración Electrónica'. Revista de Administración Pública, 179, pp.291-332.

GASCÓ, M. (2011), "Los retos de la colaboración. ¿A qué, si no a eso, pretendemos hacer frente con la interoperabilidad?", en Reforma y Democracia, no 49.

GASCÓ, M. y ROY, J. (2006), "E-Government and Multi-Level Governance: A Comparative Examination of Catalonia, Spain, and Ontario, Canada", International Journal of Electronic Government Research, 2(4), pp.57-75.

GIL-GARCÍA, J.R., CHENGALUR-SMITH, I., y DUCHESSI, P. (2007), "Collaborative eGovernment: Impediments and Benefits of Information-Sharing Projects in the Public Sector". European Journal of Information Systems, 16, pp.121-133.

GOBIERNO DE AUSTRALIA. (2006), eGovernment Initiative. Camberra, Australian Cabinet Office. GUIJARRO, L. (2007), 'Interoperability Frameworks and Enterprise Architectures in e-Government Initiatives in Europe and the United States'. Government Information Quarterly 24 (1), pp.89-101.

IDABC. (2004), European Interoperability Framework for Pan-European eGovernment Services. Bruselas, Interoperable Delivery of European eGovernment Services to public Administrations, Businesses and Citizens-Comisión Europea.

MAYER-SCHÖNBERGER, V. (2005), "The Politics of Public Safety Communication Interoperability Regulation”. Telecommunications Policy. 29, pp.831-842.

MINISTERIO DE SANIDAD. (2011), Los Servicios Web del Sistema Nacional de Salud. Madrid: Ministerio de Sanidad, en: http://www.msc.es/organizacion/sns/servWebSNS/pdf/DipticoWeb.pdf, acceso: $1 / 7 / 2011$.

SCHOLL, H. J. y KLISCHEWSKI, R. (2007), "E-Government Integration and Interoperability. Framing the Research Agenda". International Journal of Public Administration. 30, pp.889-920.

\section{Breve currículo:}

\section{J. Ignacio Criado Grande}

Profesor-investigador en el Departamento de Ciencia Política y Relaciones Internacionales de la Universidad Autónoma de Madrid. Ha sido visiting fellow en el Oxford Internet Institute, University of Oxford, así como posdoctoral visiting scholar en el Center for Technology in Government, State University of New York (SUNY at Albany). Profesor visitante de la División de Administración Pública, CIDE, México. Publicaciones sobre las interacciones entre las Tecnologías de la Información y la Comunicación y las diferentes esferas del gobierno y la administración, en revistas: Social Science Computer Review, Government Information Quarterly, Information Polity, International Journal of Electronic Governance, International Journal of Public Sector Management, Gestión y Política Pública, Reforma y Democracia o Revista de Estudios Políticos. 